\title{
Two unique presentations of Achromobacter xylosoxidans infections in clinical settings
}

\author{
Vandana Kalwaje Eshwara ${ }^{1}$, Chiranjay Mukhopadhyay ${ }^{1}$, Savitha Mohan ${ }^{1}$, Rajat Prakash ${ }^{1}$, \\ Ganesh $\mathrm{Pai}^{2}$ \\ ${ }^{1}$ Department of Microbiology, Kasturba Medical College, Manipal, Manipal University, Karnataka, India \\ ${ }^{2}$ Department of Gastroenterology, Kasturba Medical College, Manipal, Manipal University, Karnataka, India
}

\begin{abstract}
Two rare and unique infections of Achromobacter xylosoxidans are described. The first case is a novel presentation of acute necrotising pancreatitis leading to a pancreatic pseudocyst, which was treated successfully in an immunocompetent male. The second case describes a local wound infection of metastatic ductal carcinoma of the breast; the patient consequently succumbed from a pre-existing co-morbid condition. Vigilant and efficient microbiological workup and surveillance are needed to diagnose infections by this rare pathogen in clinical settings.
\end{abstract}

Key words: Achromobacter xylosoxidans, carcinoma of breast, pancreatic pseudocyst, wound infection

J Infect Dev Ctries 2011; 5(2):138-141.

(Received 04 June 2010 - Accepted 27 July 2010)

Copyright $\odot 2011$ Eshwara et al. This is an open-access article distributed under the Creative Commons Attribution License, which permits unrestricted use, distribution, and reproduction in any medium, provided the original work is properly cited.

\section{Introduction}

Achromobacter xylosoxidans, formerly called Alcaligens xylosoxidance, is a non-fermenting Gram-negative bacillus of low virulence. The recent reclassification of the bacteria denotes two subspecies namely denitrificans and xylosoxidans under the species xylosoxidans [1]. This bacterium normally inhabits aquatic sources in the environment and hospital as well as the human gut, but may cause nosocomial and community-acquired infections. Invasive infections by this bacterium in immunocompromised individuals and neonates show a high mortality [2,3]. Most infections are acquired during hospitalisation, with primary uncomplicated bacteremia, catheter-associated infections, and pneumonia being the common clinical presentations [3]. Other infections such as post-operative infections of the eye, meningitis, endocarditis and hepatobiliary infections are also reported [4-7]. We present two unique cases: in the first, Achromobacter xylosoxidans caused an infection leading to a pancreatic pseudocyst in an adult immunocompetant man. The second describes a nosocomially acquired wound infection in a woman with metastatic carcinoma of the breast.

\section{Case 1}

The first patient was a 42-year-old man who was admitted with history of sudden epigastric pain and four to six episodes of vomiting for one day. He also had continuous and nonradiating abdominal pain in the right hypochondriac region with no vomiting for last 15 days. He was on treatment for epilepsy for 12 years and hypertension for two years, and had undergone lumbosacral disc surgery five years previously. For his musculoskeletal pain, he often consumed non-steroidal anti-inflammatory drugs (NSAIDs). He denied alcohol intake or smoking.

On examination, the patient was stable with normal cardiovascular and respiratory systems. His abdomen was tender in the epigastric region and distended, with no organomegaly. Laboratory investigations revealed haemoglobin $14.6 \mathrm{mg} / \mathrm{dL}$, a total leucocyte count of $12,500 /$ cumm with $85.9 \%$ neutrophils, $7.3 \%$ lymphocytes, $5.3 \%$ monocytes and $0.9 \%$ eosinophils, and a platelet count of 1 , $50,000 /$ cumm. Other than elevated serum amylase and lipase $(780 \mathrm{IU} / \mathrm{ml}$ and $999 \mathrm{IU} / \mathrm{ml})$, the patient's blood sugar, liver and renal function tests were normal. Abdominal ultrasonography suggested acute pancreatitis.

The patient was put on conservative management and meropenem empirically. His symptoms did not improve and subsequent ultrasonography findings were suggestive of pancreatic pseudocyst that was supported by 
computed tomography (CT) of the abdomen. Purulent fluid was drained by endoscopic retrograde cholangiopancreatograpgy (ERCP), which upon culture on sheep blood agar and MacConkey agar showed a pure growth of oxidasepositive, non-fermenting, Gram-negative bacilli that was identified as Achromobacter denitrificans by API 20 NE (bioMérieux, La Balme les Grottes, France). An antimicrobial susceptibility test was performed using the Kirbey Bauer disk diffusion method and the results were interpreted using the guidelines laid by the Clinical Laboratory Standards Institute (CLSI) for non enterobacteraceae [8]. Susceptibility for cefoperazone sulbactum was tested using a disk containing $75 \mu \mathrm{g}$ of cefoperazone and $30 \mu \mathrm{g}$ of sulbactam. The zone diameter used for cefoperazone in the CLSI guideline was utilized for interpreting results for cefoperazone/sulbactam. The isolate was found susceptible to amikacin, gentamicin, tobramycin, cefotaxime, ceftazidime, ciprofloxacin, piperacillin, doxycycline, cefoperazone sulbactum, piperacillin tazobactum, and meropenem, and resistant to ampicillin, cefazolin and cefuroxime. After obtaining the antibiogram, antibiotic therapy was changed to cefoperazone sulbactum and was continued for 14 days. His post-ERCP period was unremarkable with complete recovery.

\section{Case 2}

The second patient was a 40-year-old woman, who presented with generalised weakness, loss of appetite, and breathlessness for one month. The symptom of breathlessness had increased in the last two days. She also had an ulcer on the right chest wall and axilla for seven months, for which she was receiving wound care in the same hospital. She was a known case of ductal carcinoma of the right breast diagnosed nine years previously, for which she had undergone a mastectomy followed by chemotherapy and radiotherapy. She subsequently developed a subcutaneous recurrence and metastasis for the last four years. She also had bronchial asthma.

On examination, the patient was breathless, pale, and jaundiced. Her blood pressure was $110 / 70$ $\mathrm{mm}$ of $\mathrm{Hg}$, pulse $100 / \mathrm{min}$, respiration $34 / \mathrm{min}$ and $\mathrm{SPO}_{2} 96 \mathrm{~mm} \mathrm{Hg}$. Heart sounds were normal, but she had bilateral crepitations and rhonchi. Her abdomen was grossly distended from ascites; the liver was palpable and examination revealed an irregular surface. There was a large ulcer over the right chest and another in the right axilla, from which the wound swabs were collected for bacteriological culture on the day of admission.
Abdominal ultrasonography showed metastatic liver disease and gross ascites. Chest $\mathrm{X}$ ray was suggestive of bilateral pneumonia and left pleural effusion. Laboratory investigations showed $\mathrm{Hb} 10.5 \mathrm{~g} / \mathrm{dL}$, total leucocyte count of $16,500 / \mathrm{mm}^{3}$ with $87 \%$ neutrophils and $11 \%$ lymphocytes. Liver function tests were grossly abnormal, while kidney functions were normal. Empirical antimicrobial therapy with injected levofloxacin and cefotaxime was administered along with supportive measures. On the second day of admission, she developed hypotension that was managed with dopamine and noradrenaline. On the third day, her condition worsened and she suddenly developed cardiopulmonary arrest which did not respond to resuscitation. Meanwhile, the wound specimen showed a pure and moderate growth of $A$. xylosoxidans that was susceptible to amikacin, gentamicin, tobramycin, cefotaxime, ceftazidime, ciprofloxacin, piperacillin, doxycycline, cefoperazone sulbactum, piperacillin tazobactum, and meropenem, but was resistant to ampicillin, cefazolin and cefuroxime. Following the isolation of this uncommon bacterium, overall surveillance cultures were conducted in the ward to trace the source of the bacteria. For this purpose, the unused dressing material, swabs from bed linen, rails of the bed, instruments used for dressing, saline and betadine solutions were cultured. A. xylosoxidans that had a similar antibiogram as that of the clinical isolate from the patient's wound swab was isolated from the dressing material, while other surveillance cultures remained sterile. However, the genetic relatedness of the clinical and environmental isolates could not be established because of the technological limitations at our facility. In this case, the isolate was thought to be pathogenic locally as the concomitant blood cultures remained sterile.

\section{Discussion}

A. xylosoxidans is an opportunistic pathogen responsible for various nosocomial and communityacquired infections. The source of infection is either endogenous or from a contaminated aquatic hospital environment. Mortality is high in immunocompromised patients with invasive infections [2]. Clinical presentations are diverse, including life-threatening infections such as endocarditis [3,4-7]. Our first case documents a novel presentation of an infected pancreatic pseudocyst in an immunocompetant adult by $A$. xylosoxidans, while the second case involves a wound infection acquired nosocomially from contaminated wound dressings. Both cases involve infections from this rare bacterium. 
Common organisms implicated in pancreatic infections are Escherichia coli, Enterococcus spp, Staphylococcus aureus, Klebsiella spp, Pseudomonas spp. and Proteus spp. One report of Acinetobacter associated with infected pancreatic pseudocyst is available [9], but our case is a rare presentation by $A$. xylosoxidans. The patient's gut flora could have been a putative source of infection in this case. Achromobacter is reported in some hepatobiliary and abdominal infections in the form of liver abscesses, dialysis-associated peritonitis, or severe intra abdominal infections [7,10,11], but to the best of our knowledge, infection of a pancreatic pseudocyst has never been reported. The nonfermenting, Gram-negative bacilli that are infrequently isolated in clinical microbiology laboratories face a delay in identification and add to the diagnostic dilemma because of their saprophytic nature. Often they are overlooked as contaminants. As reported earlier, these bacteria demonstrate resistance to many classes of antimicrobials [3], though in our case it was susceptible to thirdgeneration cephalosporins and aminoglycosides. Their antimicrobial resistance may further complicate therapy management and should always be guided by antibiograms. Our first case highlights the significance of uncommon non-fermenting Gram-negative bacilli in a localised infection of the pancreas.

In the second case, although $A$. xylosoxidans did not directly contribute to the case fatality, its role in wound infection cannot be underestimated, especially in severely immunocompromised patients and in those with several co-morbid illnesses. The presence of Achromobacter in the wound, either as a coloniser or as a significant pathogen in wound infection, might lead to invasive infections that could be fatal in such individuals $[2,3]$. Moreover, nosocomial acquisition of the bacteria from contaminated dressings, as evident in our report, or contaminated disinfectants and infusates, reiterate vigilant and strict infection control practices in health-care settings in the era of emerging pathogens and a large susceptible, highrisk patient population. A brief of the literature describing the acquisition of severe nosocomial infections from Achromobacter, especially in compromised hosts, supports the significance of this bacterium. In a large case series by Puthucheary et al., various nosocomial infections, including septicaemia, wound infection, otitis and meningitis by $A$. xylosoxidans, were documented mostly in hospitalised patients with serious underlying diseases who had been subjected to various invasive procedures [12]. Gomez-Cerezo et al. reported 52 episodes of nosocomial bacteremia, most commonly in patients with neoplasms. Hemodialysis units and health-care workers contaminating the intravenous catheters were found to be the sources of infections in almost $60 \%$ of patients [13]. Aisenberg et al. published a bacteremic series in cancer patients of which $33 \%$ were nosocomially acquired. Infected intravenous catheters were the sources in $25 \%$ cases while pneumonia, urinary tract infection, mucocutaneous infection and sinusitis were the other foci of bacteremia [3].

\section{Conclusion}

Our findings further expand the knowledge about the clinical spectrum of infections by the rare but important opportunistic pathogen Achromobacter. Isolation from deep-seated infections such as pancreatic pseudocysts should alert microbiologists toward appropriate identification of the bacteria and guide antimicrobial therapy to avoid complications, as the non-fermenting, Gram-negative bacteria are often overlooked as environmental contaminants. Similarly, wound infections in immunocompromised individuals by Achromobacter from contaminated wound dressings underscores the need for strict infection control practices in patient care areas.

\section{References}

1. Garrity GM, Brenner DJ, Kreig NR, Staley JT, eds (2005) Bergey's Manual of Systematic Bacteriology, 2nd ed, Springer-Verlag: New York-Berlin-Heidelberg; Vol. 2: 658-659.

2. Duggan JM, Goldstein SJ, Chenoweth CE, Kauffman CA, Bradley SF (1996) Achromobacter xylosoxidans bacteremia: report of four cases and review of the literature. Clin Infect Dis 23: 569-576.

3. Aisenberg G, Rolston K.V, Safdar A (2004) Bacteremia caused by Achromobacter and Alcaligenes species in 46 patients with cancer (1989-2003) Cancer 101: 2134-2140.

4. de-la-Torre A, López-Castillo CA, Bernal-Urrego JA, Sinisterra-Pava JP, Gómez-Marín JE (2008) Postoperative Alcaligenes xylosoxidans endophthalmitis: report of two cases. Arq Bras Oftalmol 71: 115-117.

5. Espinoza-Gómez F, Newton-Sánchez OA, Melnikov V, Virgen-González O, Unrau J (2007) Meningitis caused by Alcaligenes xylosoxidans in a patient with HIV/AIDS.Braz J.Infect Dis 11: 603-604.

6. van Hal S, Stark D, Marriott D, Harkness J (2008). Achromobacter xylosoxidans subsp. Xylosoxidans prosthetic aortic valve infective endocarditis and aortic root abscesses. J Med Microbiol 57: 525-527.

7. Asano K, Tada S, Matsumoto T, Miyase S, Kamio T, Sakurai K, Iida M (2005)

A novel bacterium Achromobacter xylosoxidans as a cause of liver abscess: three case reports. J Hepatol 43: 362-365.

8. Clinical Laboratory Standards Institute (Formerly National Committee for Clinical Laboratory Standards) (2005) Performance standards for antimicrobial disk 
susceptibility testing; fifteenth informational supplement. Approved standard M2-A8. National Committee for Clinical Laboratory Standards, Wayne, Pa.

9. Rodríguez CN, Rodríguez Morales AJ, Garcia A, Pastran B, Jimenez I (2006) Pancreatic pseudocyst infection due to multidrug resistant acinetobacter baumannii. Revista de la Sociedad Médico-Quirúrgica del Hospital de Emergencia Pérez de León 36: 5-8.

10. Melgosa M, Espinazo O, Alonso A, García Perea A, Navarro M (2004) Dialysis-Associated Alcaligenes Xylosoxidans Peritonitis:a Pediatric Case. Perit Dial Int 24: 72-75.

11. Teng SO, Ou TY, Hsieh YC, Lee WC, Lin YC, Lee WS (2009) Complicated intra abdominal infection caused by extended drug resistant Achromobacter xylosoxidans. J Microbiol Immunol Infect 42: 176-180.

12. Puthucheary SD, Ngeow YF (1986) Infections with Achromobacter xylosoxidans. Singapore Med J 27: 58-62.
13. Gómez-Cerezo J, Suárez I, Ríos JJ, Peña P, García de Miguel MJ, de José M, Monteagudo O, Linares $P$, Barbado-Cano A, Vázquez JJ (2003) Achromobacter xylosoxidans bacteremia: a 10-year analysis of 54 cases. Eur J Clin Microbiol Infect Dis 22: 360-363.

\section{Corresponding author}

Dr. K E Vandana

Associate Professor

Department of Microbiology

Kasturba Medical College, Manipal - 576104

Karnataka, India

Telephone: 918202922322 (O); 91 9902206009(cell); Fax: 91 8202571927

Email: vandanake@gmail.com

Conflict of interests: No conflict of interests is declared. 